\title{
140. On Probabilities of Non-Paternity with Reference to Consanguinity. II
}

\author{
By Yûsaku Komatu and Han NishimiYa \\ Department of Mathematics, Tokyo Institute of Technology and \\ Department of Mathematics, Shibaura Institute of Technology \\ (Comm. by T. Furuhata, M.J.A., Dec. 12, 1959)
}

3. Non-paternity of a putative man related with the mother but not with the father. We now proceed to the case where a putative man and the mother of a child have antecedants of $\mu$ th and $\nu$ th generations respectively in common while the true father of the child is in no consanguineous relation with them. The probability of the triple which consists of a putative man $A_{a b}$ and a mother-child combination $(\alpha \beta ; \xi \eta)$ under the imposed relationship is given by

$$
\bar{A}_{\alpha \beta} V\left(\alpha \beta ; \xi \eta \mid \sigma_{\mu, \nu+1}\right)=\sum_{\Omega} \sigma_{\mu \nu}(\alpha b, \alpha \beta),
$$

where the range of summation is, as before, the set

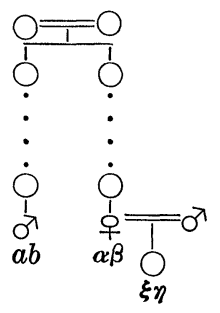
$\Omega=\Omega(\alpha \beta ; \xi \eta)$ of types $A_{a b}$ which together with $A_{\alpha \beta}$ can not produce $A_{\xi \eta}$. Now fortunately here also, it can be shown directly that there exists a remarkable identity

$$
V\left(\alpha \beta ; \xi \eta \mid \sigma_{\mu, \nu+1}^{\circ}\right)=\left(1-2^{-\lambda+1}\right) V(\alpha \beta ; \xi \eta)
$$

provided $\lambda=\mu+\nu-1>1$, while for the exceptional value $\lambda=1$, i.e. $\mu=$ $\nu=1$ we have

$$
V\left(\alpha \beta ; \xi \eta \mid \sigma_{1,2}^{\circ}\right)=\frac{1}{4} V(\alpha \beta ; \xi \eta) .
$$

Consequently, subsequent arguments can be economized and really reduced to those in the ordinary case without any consanguinity. The final result for the total probability of non-paternity in the present case is given by

$$
P\left(\sigma_{\mu, \nu+1}\right)=\left\{\begin{array}{lc}
\frac{1}{4} P & (\mu=\nu=1), \\
\left(1-2^{-\lambda+1}\right) P & (\lambda=\mu+\nu-1>1) .
\end{array}\right.
$$

The decrement of $P\left(\sigma_{\mu, \nu+1}\right)$ compared with $P$ as well as its behavior as $\lambda \rightarrow \infty$ is quite similar as in the previous case. In particular, we now have

and

$$
P-P\left(\sigma_{\mu, \nu+1}\right)= \begin{cases}\frac{3}{2}\left(P-P\left(\sigma_{\mu, \nu+1}^{\alpha \lambda}\right)\right) & (\mu=\nu=1), \\ 2\left(P-P\left(\sigma_{\mu, \nu+1} \sigma^{\top}\right)\right) & (\mu+\nu>2)\end{cases}
$$

$$
P\left(\sigma_{\mu, \nu+1}\right)<P\left(\sigma_{\mu, \nu+1}\right)<P\left(\sigma_{\mu \nu ; 1}\right)<P \quad(\mu+\nu \geqq 2) .
$$

4. Illustrative examples. The general results obtained in the 
previous sections will be illustrated by means of human blood groups.

The $M N$ blood group offers the simplest example. In fact, we have only to specialize the general formulas by putting $m=2$. Denoting, as usual, by $s$ and $t$ the distribution probabilities of the genes $M$ and $N$ respectively, we get for the total probabilities of non-paternity considered the following formulas:

$$
\begin{array}{ll}
P_{M N}\left(\sigma_{\mu \nu ; 1}\right)=P_{M N}-2^{-\lambda-1} s t(1-4 s t)=\left(1-2^{-\lambda}\right) P_{M N}+2^{-\lambda-1} s t(1+2 s t), & \\
P_{M N}\left(\sigma_{\mu, \nu+1}\right)=\left(1-2^{-\lambda}\right) P_{M N}, & (\mu=\nu=1), \\
P_{M N}\left(\sigma_{\mu, \nu+1}\right)= \begin{cases}\frac{1}{4} P_{M N} & (\mu+\nu>2) . \\
\left(1-2^{-\lambda+1}\right) P_{M N} & p+\nu\end{cases}
\end{array}
$$

Here $P_{M N}$ denotes the ordinary probability of non-paternity without any consanguinity which is equal to

$$
P_{M N}=s t(1-s t) \text {. }
$$

The $A B O$ blood group involves a recessive gene. The general discussion stated in the previous section must be accordingly modified, since now the decision of consistency is to be based upon phenotypes. In fact, by virtue of the presence of the recessive gene $O$, the range $\Omega$ concerning phenotypes diminishes strictly in comparison with the sum of the constituting $\Omega$ 's concerning genotypes unless the child has the type $A B$. By following the above arguments thus modified, it is shown that the total probabilities are given by the formulas:

$$
\begin{aligned}
& P_{A B O}\left(\sigma_{\mu \nu ; 1}\right)=\left(1-2^{-\lambda}\right) P_{A B O}+2^{-\lambda} p q r(1+2 r), \\
& P_{A B O}\left(\sigma_{\mu, \nu+1}\right)=\left(1-2^{-\lambda}\right) P_{A B O}, \\
& P_{A B O}\left(\sigma_{\mu, \nu+1}\right)= \begin{cases}\frac{1}{4} P_{A B O} & (\mu=\nu=1), \\
\left(1-2^{-\lambda+1}\right) P_{A B O} & (\mu+\nu>2)\end{cases}
\end{aligned}
$$

where $p, q$ and $r$ denote, as usual, the distribution probabilities of the genes $A, B$ and $O$, respectively, and $P_{A B O}$ the ordinary probability of non-paternity which is equal to

$$
P_{A B O}=p(1-p)^{4}+q(1-q)^{4}+p q r^{2}(3-r) \text {. }
$$

The decrease of the probabilities based on the presence of consanguinity is perceived here also. Namely, it is shown that we have a system of inequalities

$$
P_{A B O}\left(\sigma_{\mu, \nu+1}^{\circ}\right)<P_{A B O}\left(\sigma_{\mu, \nu+1}\right)<P_{A B O}\left(\sigma_{\mu \nu ; 1}\right)<P_{A B O} .
$$

In particular, the last inequality may be verified as follows:

$$
\begin{aligned}
& 2^{\lambda}\left(P_{A B O}-P_{A B o}\left(\sigma_{\mu \nu ; 1}\right)\right)=p(1-p)^{4}+q(1-q)^{4}-p q r\left(1-r+r^{2}\right) \\
= & (1-r) r^{4}+p q\left((p-q)^{2}(1+2 r)+r^{2}(5+2 r)+p q(1+3 r)\right)>0 .
\end{aligned}
$$

Here the use is made of the identity $p+q+r=1$.

It is noted here, by the way, that the absence of the gene $O$ causes a sort of discontinuity of the probabilities with respect to $r$. This is a phenomenon which has appeared also in case of non-consanguinity. In fact, if the gene $O$ is known to be entirely absent, the 
mode of inheritance of the $A B O$ group becomes quite same as that of the $M N$ group. Hence, for instance, the probability $P_{A B O}\left(\sigma_{\mu \nu ; 1}\right)$ for such an extreme distribution is given by

$$
P_{A B O}\left(\sigma_{\mu \nu ; 1}\right)=p q(1-p q)-2^{-\lambda-1} p q(1-4 p q)
$$

while, by merely putting $r=0$, the value obtained in the generic case becomes

$$
\left(1-2^{-\lambda}\right) p q(1-3 p q)
$$

which is different from the former true value. More precisely, the value of the latter is smaller by $2\left(1-2^{-\lambda-1}\right) p^{2} q^{2}+2^{-\lambda-1} p q$ than that of the former. Such discontinuity appears also for other kinds of probabilities considered above.

Such discontinuity appears also between $Q$ and $M N$ blood groups by virtue of the recessive gene $q$ involved in the former. However, it does not appear between $A B O$ and $Q$ blood groups. In fact, the probabilities for the $Q$ group may be obtained from those for the $A B O$ group by simply putting $(p, q, r)=(u, 0, v), u$ and $v$ being the distribution probabilities of the genes $Q$ and $q$, respectively. We thus get

with

$$
\begin{aligned}
& P_{Q}\left(\sigma_{\mu \nu ; 1}\right)=\left(1-2^{-\lambda}\right) P_{Q}, \quad P_{Q}\left(\sigma_{\mu, \nu+1}\right)=\left(1-2^{-\lambda}\right) P_{Q}, \\
& P_{Q}\left(\sigma_{\mu, \nu+1}\right)=\left\{\begin{array}{lr}
\frac{1}{4} P_{Q} & (\mu=\nu=1), \\
\left(1-2^{-\lambda+1}\right) P_{Q} & (\lambda=\mu+\nu>2)
\end{array}\right.
\end{aligned}
$$

$$
P_{Q}=u v^{4} \text {. }
$$

5. Absolute non-paternity. The discussions until now have been concerned with proving of non-paternity of a man against a motherchild combination. But there are cases where the non-paternity proof is possible with no regard to the type of mother. This constitutes the absolute non-paternity.

Among three kinds of consanguinity considered in the previous sections, the last two, after disregarding the type of mother, become coincident for our present problems of absolute non-paternity. Consequently, we now have to distinguish two cases.

We begin with the case where a putative man can prove his nonpaternity against a child who is in no consanguineous relation with him but whose parents have $\mu$ th and $\nu$ th antecedants in common. The distribution probability of a child under the imposed condition is given by

$$
\bar{A}_{\mu \nu ; 1}(i i)=\bar{A}_{i i}+2^{-\lambda-1} i(1-i), \quad \bar{A}_{\mu \nu ; 1}(i j)=\bar{A}_{i j}-2^{-2} i j,
$$

valid for any $\mu$ and $\nu$. On the other hand, the probability that a man can prove his non-paternity against a given child of $A_{\xi \eta}$ is given by

$$
V(\xi \eta)=\sum_{\Omega} \bar{A}_{a b}
$$

where the summation extends over the set $\Omega=\Omega(\xi \eta)$ of types $A_{a b}$ which are absolutely inconsistent with $A_{\xi \eta}$. It is expressed by the formula 


$$
V(i i)=(1-i)^{2}, \quad V(i j)=(1-i-j)^{2} .
$$

Consequently, the total absolute probability of non-paternity in this case is given by

$$
\begin{aligned}
C\left(\sigma_{\mu \nu ; 1}\right) & =\sum \bar{A}_{\mu \nu ; 1}(\xi \eta) V(\xi \eta)=\left(1-2^{-i-1}\right) C+2^{-\lambda-1}\left(1-2 S_{2}+S_{3}\right) \\
& =C+2^{-\lambda-1}\left(2 S_{2}-3 S_{3}-2 S_{2}^{2}+3 S_{4}\right)
\end{aligned}
$$

where $C$ is the corresponding probability in the ordinary case which is equal to

$$
C=1-4 S_{2}+4 S_{3}+2 S_{2}^{2}-3 S_{4}
$$

Compared with $\bar{A}_{\xi \eta}$, the probability $\bar{A}_{\mu \nu ; 1}(\xi \eta)$ increases or decreases according to $A_{\xi \eta}$ to be homozygous or heterozygous. But the above formula shows that these deviations cause as the whole the increase of the total probability. In fact, we get

$$
2^{\lambda+1}\left(C\left(\sigma_{\mu \nu ; 1}\right)-C\right)=\Sigma^{\prime} i j\left(2(i+j)(1-i-j)+i^{2}+j^{2}\right)
$$

which remains always positive except a trivial case of a single gene.

We now proceed to the case where a putative man and a child in question have $\mu$ th and $(\nu+1)$ th antecedants in common. Similar arguments as above lead to the total probability of non-paternity in this present case. Namely, we have the expression

or alternatively

$$
C\left(\sigma_{\mu, \nu+1}\right)=\sum_{\xi \eta} \bar{A}_{\xi \eta} \sum_{\Omega} \sigma_{\mu, \nu+1}(a b, \xi \eta)
$$

$$
C\left(\sigma_{\mu, \nu+1}\right)=\sum_{a b} \sum_{\xi \eta} \sigma_{\mu, \nu+1}(a b, \xi \eta) V(\xi \eta) .
$$

The final formula is then given by the formula

$$
C\left(\sigma_{\mu, \nu+1}\right)=\left(1-2^{-\lambda}\right) C \quad(\lambda=\mu+\nu-1 \geqq 1) .
$$

The decrease by virtue of the presence of consanguinity is evident.

In conclusion, we shall illustrate the results by means of human blood groups. A modification similar as above will be required according to the presence of a recessive gene. The final formulas are as follows:

$$
\begin{array}{ll}
C_{M N}\left(\sigma_{\mu \nu ; 1}\right)=C_{M N}+2^{-\lambda} s t(1-2 s t), & C_{A B O}\left(\sigma_{\mu \nu ; 1}\right)=C_{A B O}+2^{-\lambda} p q r(1-2 r) ; \\
C_{M N}\left(\sigma_{\mu, \nu+1}\right)=\left(1-2^{-\lambda}\right) C_{M N}, & C_{A B O}\left(\sigma_{\mu, \nu+1}\right)=\left(1-2^{-\lambda}\right) C_{A B O},
\end{array}
$$
the $C$ 's denoting the ordinary probabilities given by

$$
C_{M N}=2 s^{2} t^{2}, \quad C_{A B O}=4 p q r^{2} \text {. }
$$

Though, as shown above, the inequality $C\left(\sigma_{\mu \nu ; 1}\right)>C$ holds generally in genotypic decision, it is not necessarily true in phenotypic decision. In fact, the difference $C_{A B O}\left(\sigma_{\mu \nu ; 1}\right)-C_{A B O}=2^{-2} p q r(1-2 r)$ is positive for $r<1 / 2$ but negative for $r>1 / 2$. However, it may be noted that the difference

$$
C_{A B O}\left(\sigma_{\mu \nu ; 1}\right)-C_{A B O}\left(\sigma_{\mu, \nu+1}\right)=2^{-\lambda} p q r(1+2 r)
$$

remains always positive provided every gene is really present.

The phenomenon of discontinuity between these groups appears here also by virtue of the presence of the recessive gene. 